\title{
Pathology and Pathophysiology of Pulmonary Manifestations in Leptospirosis
}

\author{
Marisa Dolhnikoff ${ }^{1}$, Thais Mauad ${ }^{1}$, Eduardo P. Bethlem ${ }^{3}$ and Carlos Roberto Ribeiro Carvalho ${ }^{2}$ \\ ${ }^{1}$ Department of Pathology, School of Medicine, University of São Paulo; ${ }^{2}$ Pulmonary Division, Hospital das Clinicas - Heart Institute (InCor), \\ School of Medicine, University of São Paulo; São Paulo, SP; ${ }^{3}$ Division of Pulmonary Medicine, Federal University of Rio de Janeiro State \\ (UNI-RIO); Rio de Janeiro, RJ, Brazil
}

\begin{abstract}
Leptospirosis is a re-emerging zoonosis occurring as large outbreaks throughout the world caused by Leptospira interrogans. The incidence of pulmonary involvement in leptospirosis has been reported to be increasing in the last years, affecting up to $70 \%$ of the patients. Alveolar hemorrhage presented as dyspnea and hemoptysis is the main pulmonary manifestation. The emergence of massive hemoptysis and acute respiratory distress syndrome has characterized the recent changes reported in the clinical patterns of leptospirosis. The pulmonary involvement has been emerged as a serious life threat, becoming the main cause of death due to leptospirosis in some countries. In this review we present the main clinical and pathological manifestations of pulmonary involvement in leptospirosis, with special focus on recent data concerning the pathophysiological mechanisms underlying lung injury.

Key-Words: Leptospirosis, pulmonary hemorrhage, diffuse alveolar damage.
\end{abstract}

Leptospirosis is a re-emerging zoonosis occurring as large outbreaks throughout the world caused by Leptospira interrogans. It affects humans in both urban and rural areas, and in temperate and tropical climates [1]. Although considered to be the most geographically widespread zoonosis, it is a sporadic disease, restricted to risk exposure associated with specific occupational groups, such as farmers, miners, and abattoir and sewer workers, as well as with recreational activities. In Latin America, leptospirosis has been recognized as a major urban pathogen causing epidemics. In Brazil, for example, approximately 10,000 cases are reported each year in all major cities. Overall mortality is $10 \%-15 \%$ among outbreak cases [2].

Leptospirosis occurs when humans come into direct contact with the urine or tissue of carrier mammals (usually rats - Rattus norvegicus, but mice, sheep, cattle, pigs, dogs, racoons, goats, marsupials, and bats have been reported as possible reservoirs), or indirectly through contaminated water, soil, or vegetation. The usual portal of entry is abraded skin or intact mucous membranes. The spirochetes then travel to the liver where they reproduce [3,4].

Human infection by pathogenic Leptospira may present variable clinical manifestations ranging from subclinical infection with undifferentiated febrile illness to jaundice, renal failure, and potentially lethal pulmonary disease [5]. Leptospirosis is typically a biphasic illness (anicteric), but a fulminant disease (icterohaemorrhagic form, Weil's Disease) can be found in $5-10 \%$ of all patients. Fatalities typically arise from renal, cardiac, or respiratory failure [4].

Received on 12 September 2006; revised 20 December 2006.

Address for correspondence: Dr. Marisa Dolhnikoff. Departamento de Patologia. Faculdade de Medicina da Universidade de São Paulo. Av. Dr. Arnaldo, 455 - Sala 1155. Zip code: 01246-903. São Paulo, SP, Brazil. Fax: 55-11-3062-8098, Phone: 55-11-3066-7377, email: maridol@usp.br.

Financial support: Conselho Nacional de Desenvolvimento Científico e Tecnológico (CNPq).

The Brazilian Journal of Infectious Diseases

2007;11(1):142-148 (C) 2007 by The Brazilian Journal of Infectious Diseases and Contexto Publishing. All rights reserved.
In the biphasic illness the initial acute or septicemic phase is characterized by bacteremia that typically lasts about one week. Most of the cases present with a febrile illness of sudden onset. Fever, chills, headache, severe myalgia, conjunctival suffusion, anorexia, nausea, vomiting, and prostration usually characterize acute leptospirosis. A substantial proportion of people infected by Leptospira may have subclinical disease or very mild symptoms, and do not seek medical attention [5]. In this leptospiremic phase, leptospires can be found in the blood and cerebrospinal fluid [3]. The resolution of symptoms may coincide with the second or immune phase, when circulating immunoglobulin M (IgM) antibodies begin to be produced, accompanied by excretion of spirochetes in the urine. However, fever may recur after a remission of 3 to 4 days, producing a biphasic illness.

Weil's disease represents only the most severe form of the illness. This syndrome can develop after the acute phase as the second phase of a biphasic illness, or can simply present as a single, progressive illness. It is characterized by high fever, intense jaundice, bleeding, renal and pulmonary dysfunction, neurologic alterations, and cardiovascular collapse, with a variable clinical course [6,7].

\section{Pulmonary Involvement in Leptospirosis}

The incidence of pulmonary involvement in Leptospirosis varies from $20 \%$ to $70 \%$, and has been reported to be increasing in the last years. Whereas pulmonary manifestations were reported to be less prominent in the past, with hemoptysis being observed in $3-25 \%$ of patients and chest pain in $10 \%$, epidemics of leptospirosis wherein pulmonary manifestations dominated the clinical pictures have been reported since the late 1980s [8-13]. Alveolar hemorrhage presented as dyspnea and hemoptysis is the main pulmonary manifestation in leptospirosis; it may vary from mild to severe, severity being related to mortality [5,14]. In a cohort of 26 Spanish patients, 17 had respiratory symptoms (65\%), and three patients died of multiorgan failure following acute respiratory distress syndrome (ARDS) [3]. 
Pulmonary involvement in leptospirosis is reported to be poorly recognized in regions where it is endemic. A prospective study was carried out to identify febrile patients exposed to Leptospira in urban and rural areas in Iquitos, Peru. Of 633 patients presenting with fever and jaundice, 321 (50.7\%) had anti-leptospiral IgM antibodies of high titres. Of the 321 patients with leptospirosis, 189 were from urban location and 132 from rural areas. In the rural community, no cases of severe pulmonary leptospirosis occurred. Seven patients (3.7\%) with history of only urban exposure to leptospires had severe pulmonary manifestations; of these, five patients died, four of them due to pulmonary hemorrhage and one due to ARDS and multiorgan failure. This study demonstrated the underdiagnosis of leptospirosis and the underrecognition of severe pulmonary involvement in this disease in a region of high endemicity [15].

Human leptospirosis is one of the main urban endemics/ epidemics in Brazil. Its incidence has grown in the last three decades, especially after floods caused by summer rains. Recent changes in the clinical patterns of leptospirosis has been reported in Brazil, expressed by the emergence of massive hemoptysis and acute respiratory distress syndrome, or both conditions associated. The pulmonary involvement emerged as a serious life threat, becoming the main cause of death due to leptospirosis in that country [16].

In São Paulo, the Central Institute of the Hospital das Clinicas, a tertiary University Hospital, with about 950 beds (being 150, Intensive Care Unit - ICU beds), admitted 264 patients with leptospirosis between January 1991 and June 2001. This population presented a median age of 33 years old (9 to 77 years), being 223 men (85.4\%), and a lethality rate of $10.0 \%$ (26 patients died). The median duration of stay at the hospital was 10 days (zero to 42 days). About $60 \%$ of these patients were admitted to the ICU for at least one day and $10 \%$ needed mechanical ventilation [7].

\section{Clinical Manifestations}

Pulmonary symptoms occur in both anicteric and icteric forms of the disease, ranging from chest pain, cough, dyspnea, and hemoptysis, to acute respiratory distress syndrome $[4,5,14,17]$. The severity of respiratory disease is unrelated to the presence of jaundice. Intra-alveolar hemorrhage is detected in the majority of patients, even in the absence of overt pulmonary symptoms, although it may be severe enough to cause death [18]. Few cases of leptospiral pneumonia have also been reported $[19,20]$.

Hemoptysis occurred in $50 \%$ of 168 cases in China between 1959 and 1960 [21] and in 13\% of 115 patients hospitalized with severe leptospirosis in Brazil [22]. In the Spanish series of 26 patients with leptospirosis, 17 had respiratory symptoms, such as dyspnea (seven patients); hemoptysis with bloodstained sputum (seven patients); cough (six patients), and pleuritic chest pain (five patients) [3].

Severe pulmonary form of leptospirosis (SPFL) is the most severe clinical manifestation of leptospirosis. Respiratory symptoms usually appear between the fourth and sixth day of disease and may lead to death in less than 72 hours. Mortality rates can be as high as 30 to $60 \%$. Profuse lung hemorrhage dominates the clinical presentation [23]. In four patients with SPFL described in Brazil, three died in less than 48 hours after onset of the first respiratory signs. Leptospiral antigen was detected in lung tissue by immunohistochemistry in all three patients, suggesting that the microorganism, or its byproducts, exert a local direct destructive action. Autopsy showed extensive areas of pleuropulmonary hemorrhage, with a microscopic picture of hemorrhagic infiltrates involving alveoli and septa. The three patients also presented thrombocytopenia, a common finding in patients with severe leptospirosis [23-25].

In Buenos Aires, Argentina, 276 cases of leptospirosis were diagnosed between 1900 and 1999. No severe pulmonary hemorrhage was detected in these patients. During 2000 and 2001, a total of 93 cases were reported in the same area, including an outbreak of 47 cases in March 2001. Four patients died with suspected leptospirosis, three of them with confirmed diagnosis. Two of these patients died due to pulmonary hemorrhage, characterizing the first report of this clinical form of presentation in the metropolitan area of Buenos Aires [26].

Pulmonary hemorrhage occurred in 14 of 75 patients (19\%) in the Seychelles, causing the deaths of six patients [27]. In a 1987 epidemic in Korea, 37 of 93 patients (40\%) with leptospirosis had hemoptysis, massive in all 5 patients who died [28].

\section{Prognostic Factors}

Different variables have been reported to be associated with poor prognosis in patients with leptospirosis and pulmonary involvement. Alveolar infiltrates on chest radiographs and dyspnea have been shown to be poor respiratory prognostic indicators in severe leptospirosis, associated with mortality $[5,29]$. In their cohort of 26 patients, Garcia et al found that cigarette smoking was a risk factor for the development of respiratory involvement in leptospirosis. From the three patients that died, two were heavy smokers [3]. It is suggested that smoking can favor the development of pulmonary hemorrhage by increasing the permeability of lung capillaries, damaging alveolar basement membrane, and increasing the local inflammatory response $[3,30]$.

Severe pulmonary hemorrhage has been associated with high mortality rates. In a prospective study of 42 patients with leptospirosis and acute lung injury, who were mechanically ventilated, 19 patients survived (45\%), and 23 (55\%) died, 21 because of multiorgan failure and massive pulmonary hemorrhage, and two because of nosocomial infection. Three variables were independently associated with mortality in these patients: hemodynamic disturbance, serum creatinine level $>265.2 \mu \mathrm{mol} / \mathrm{L}$, and serum potassium level $>$ $4.0 \mathrm{mmol} / \mathrm{L}$. The authors suggest that these observations can be used to identify factors associated with mortality early in the course of severe respiratory failure in leptospirosis [31]. 
In a series of 46 patients with acute respiratory distress syndrome in Thailand, tropical diseases such as malaria and leptospirosis were identified as risk factors for developing the syndrome. From the 46 patients, 4 had leptospirosis and 3 died [32].

\section{Radiographic Findings}

Radiography of patients with pulmonary involvement in leptospirosis generally reveals diffuse small opacities which may be widely disseminated or which may coalesce into larger areas of consolidation, with increasing severity of symptoms [18].

In a series of 58 patients with leptospirosis, 37 (64\%) had pulmonary radiographic findings. Three radiographic patterns were evident in these patients: small nodular densities (21 patients, 57\%); confluent areas of consolidation (6 patients, 16\%); and diffuse, ill-defined, ground-glass density (10 patients, 27\%). Serial radiographs showed a tendency for the nodular pattern to be followed by confluent consolidation and/or ground-glass density. Abnormalities were bilateral, non-lobar in all cases, and had a marked tendency toward peripheral predominance [33].

The high-resolution computed tomography (HRCT) findings were reviewed in five patients with diffuse pulmonary hemorrhage caused by leptospirosis. All patients presented a predominantly HRCT picture of extensive, bilateral groundglass opacities involving all lobes. Four patients had areas of consolidation. Two patients had peripheral airspace nodules. One patient had poorly defined centrilobular nodular opacities in the upper lobes. Two patients had small bilateral pleural effusions. All five patients had chest radiographs showing extensive bilateral airspace consolidation and poorly defined ground-glass opacities involving mainly the lower lung. The HRCT was superior to chest radiography in demonstrating the extent of ground-glass opacities, thus showing the diffuse nature of the abnormalities. In one patient who died, comparison of the HRCT with the histological findings showed that the ground-glass opacities, airspace consolidation, and airspace nodules were caused by alveolar hemorrhage [34].

\section{Histological Findings and Pathophysiology in Human Pulmonary Involvement in Leptospirosis}

Pulmonary congestion and hemorrhage are common histological findings in human pulmonary involvement in leptospirosis (Figures 1 and 2). Although infiltration of alveolar spaces by monocytes and neutrophils occurs, inflammatory infiltrates are generally not prominent. Other findings, including pulmonary edema, fibrin deposition, and proliferative fibroblastic reactions, are frequent [4,35]. Hyaline membrane formation may occur, characterizing a picture of diffuse alveolar damage [14,32,36] (Figures 3 and 4). Leptospires may be seen within endothelial cells in interalveolar septa, and attached to capillary endothelial cells [37].

The pathogenesis of lung abnormalities in leptospirosis is presently under investigation. Two main mechanisms of leptospirosis pathogenesis are suggested: a toxin-mediated mechanism and/or the immune responses of the host. A toxinmediated capillary vasculitis is believed to cause lung hemorrhage [4]. Lung tissue in patients with leptospirosis usually shows much lower numbers of leptospires than liver and blood counts, suggesting that pulmonary abnormalities may be due to exposure of circulating toxins produced by the pathogen at distant sites such as the liver [5]. The multiorgan dissemination of leptospires is probably a result of its rapid cell translocation. It has been proposed that the ability of Leptospira to pass through cells may not be as important as the rate at which they penetrate them. This would facilitate rapid dissemination, before cell barriers or circulating immune cells could inhibit them [38]. Leptospires seem to attach themselves directly to the cells, initiating cellular injury. Outer membrane proteins (OMP) are possible toxins related to leptospirosis pathogenesis. Spirochetal bacteria possess two membranes and the proteins present in the outer membrane are at the site of interaction with host tissue and the immune system [39]. Other possible toxins under investigation include membrane glycoproteins [40], hemolysin [41] and lypopolysaccharides [42]. Peptidoglycans from pathogenic leptospires are among the molecules that can directly activate vascular endothelial cells to increase their adhesiveness for neutrophilic granulocytes, and may be involved in the mechanism of local and systemic inflammation in leptospirosis [43]. Cytokines, like tumor necrosis factor alpha (TNF- $\alpha$ ), may also be involved in the pathogenesis of leptospirosis [44]. Significant higher levels of circulating TNF- $\alpha$ are associated with severe leptospiral infection with kidney, liver and lung involvement as compared with patients without these complications [7].

Nicodemo et al studied the lung tissue of 12 patients that died of leptospirosis. Pulmonary hemorrhage alone or associated with gastrointestinal bleeding was the cause of death in eight patients. Light microscopy showed edema of the intra-alveolar septa; a mild-to-moderate inflammatory infiltrate, with a predominance of macrophages, lymphocytes and plasma cells; endothelial tumefaction; and alveolar hemorrhage. Leptospiral antigen was detected by immunohistochemistry on the luminal surface of the endothelium and in the cytoplasm of the endothelial cells of septal capillaries. Although the quantification of leptospiral antigen did not correlate with the intensity of the lesions, the authors suggest that the detection of intact leptospires and of granular antigenic material in capillary endothelial cells indicates that the pneumopathy is directly triggered by leptospires and/or by their toxic products. Fibrin was observed in the lumen of septal capillaries, in the alveolar lumen, and on the surface of the alveoli, characterizing a diffuse alveolar damage. There was no evidence of thrombus formation or of disseminated intravascular coagulation. The electron microscopy showed capillary disease in areas free from hemorrhage, characterized by tumefaction of endothelial cells, increased number of pinocytotic vesicles, presence of cytoplasm giant dense bodies, and emission of pseudopods. 
Figure 1. Photomicrograph of lung parenchyma showing diffuse alveolar hemorrhage. The parenchyma architecture is preserved. Note the alveolar spaces filled with blood. HEx50.

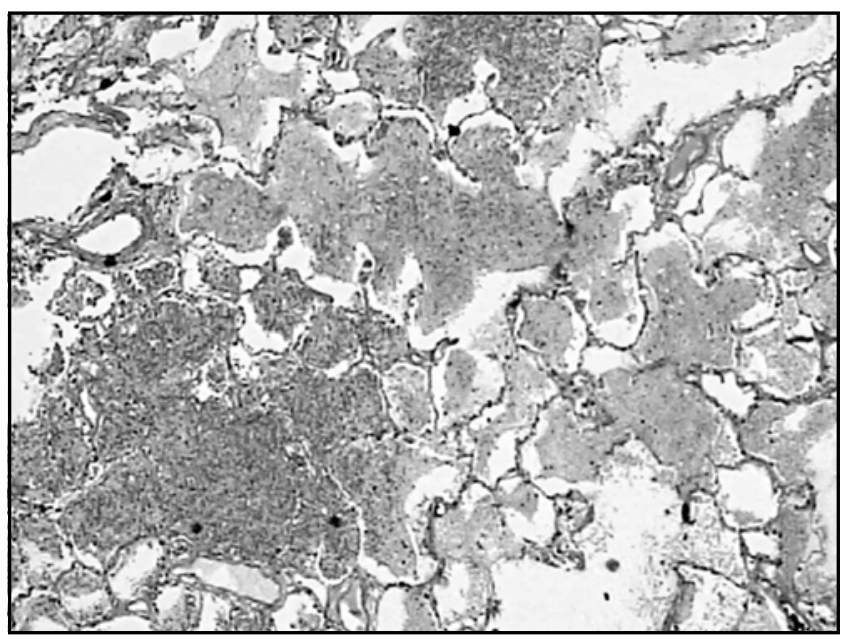

Figure 3. The lung parenchyma shows a picture of diffuse alveolar damage, characterized by hyaline membranes (arrow) and alveolar edema $(*)$, adjacent to areas of alveolar hemorrhage (top left). HE x100.

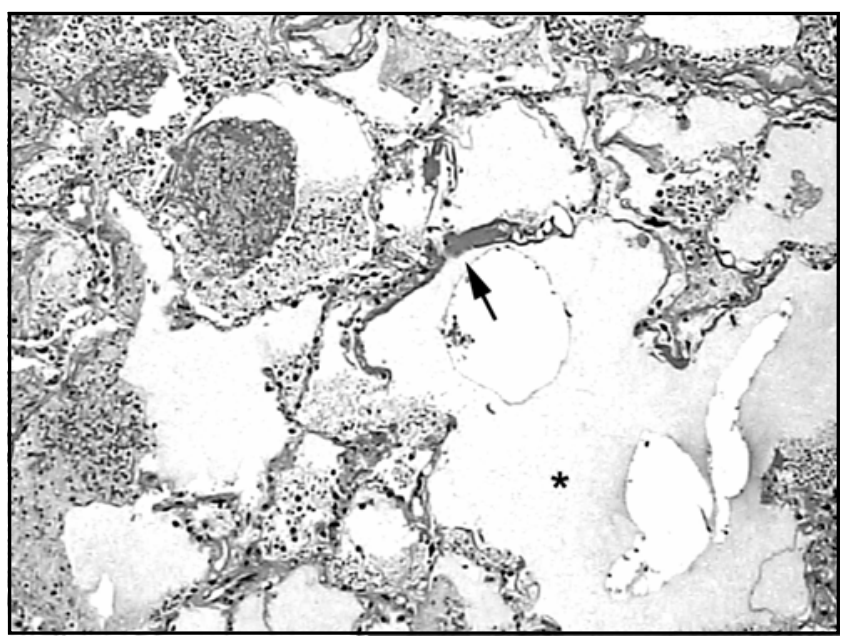

Isolated or aggregated platelets were detected in the lumen of septal capillaries, and adhering to endothelial cells, with morphological changes suggestive of platelet activation. Various mechanisms could be implicated in platelet activation, such as mechanisms related to endothelial cells, and action of bacterial endotoxins. Lipopolysacharides and cytokines could also stimulate endothelial cells and alter their equilibrium to a pro-coagulant state [37].

Besides a toxin-mediated process, an indirect pathogenic mechanism mediated by the host immune response to infection is also suggested [5]. Leptospires immunity depends on the production of circulating antibodies against the pathogen's
Figure 2. Alveolar hemorrhage in leptospirosis. The photomicrograph shows a high power view of alveolar septa. There is no evident inflammation, a common finding in pulmonary involvement in leptospirosis. HE x400.

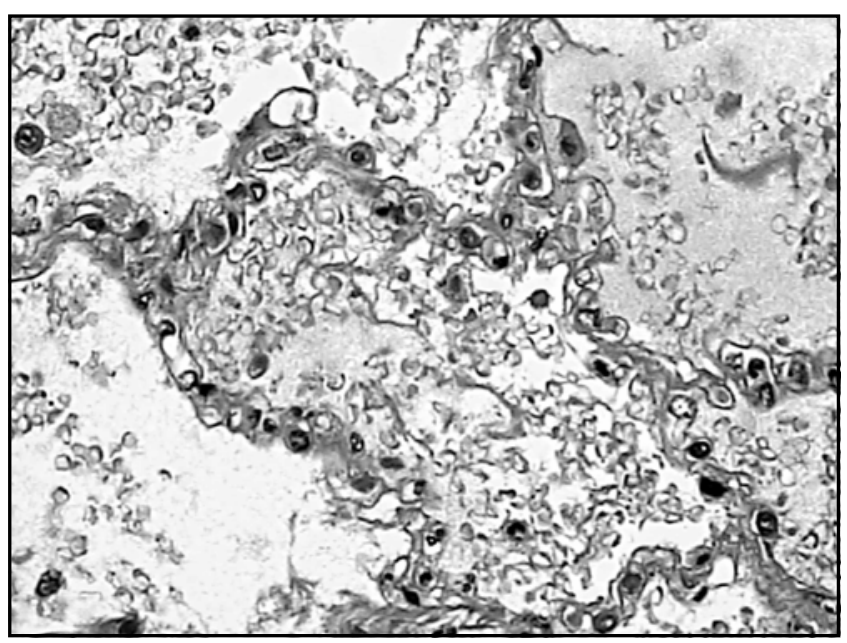

Figure 4. Hyaline membranes lining the alveolar septa in a patient with ARDS related to leptospirosis. HE x200.

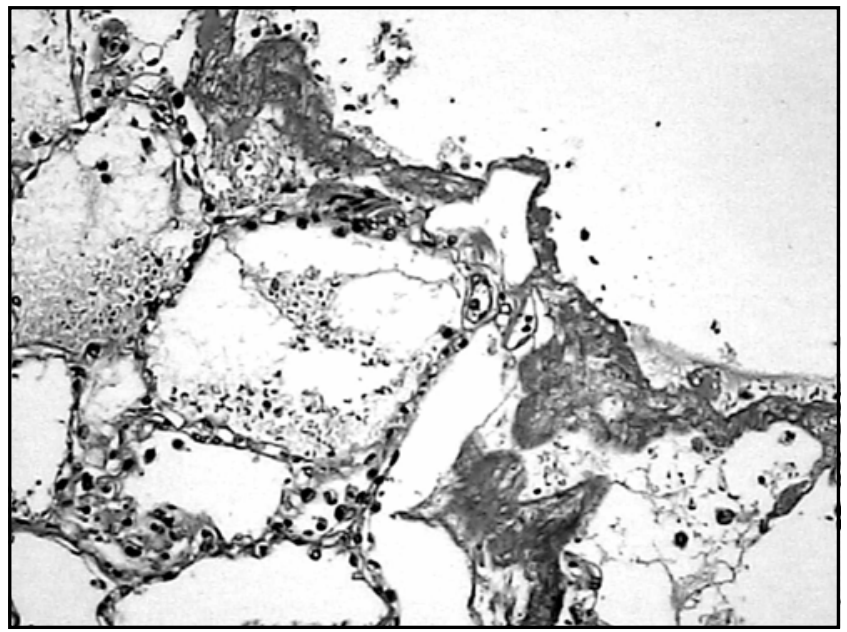

lipopolysaccharide (LPS). Interestingly, it was recently demonstrated that leptospires LPS activates cells through a toll-like receptor 2 (TLR-2) and CD14 dependent mechanism. This activation of the innate immune system is certainly the initiating event in antibody generation [45].

The second stage of acute leptospirosis is also referred to as the immune phase, in which the disappearance of the organism from the bloodstream coincides with the appearance of antibodies. Immune-mediated disease has been proposed as one factor influencing the severity of the disease [18]. In a Brazilian study, 35 hospitalized patients with Weil's disease had five blood samples drawn, from the 15th day to the 12th 
month of symptoms, for ELISA-IgM, -IgG and -IgA specific antibody detection. Patients with an IgG titer of $400(n=13)$ or more had more severe pulmonary hemorrhage and renal function damage compared with patients with an IgG titer of less than $400(n=22)$, suggesting that the severity of Weil's disease may be associated with the intensity of the humoral immune response to leptospire [46].

Recently, whole genome sequencing of the Leptospira interrogans serogroup interrogans [47] and lai [48] identified many genes with a potential role in disease pathogenesis. Genes involved in host cell membrane degradation and encoding proteases, which could participate in the mechanisms of endothelial damage, have been sequenced. Furthermore, in the serovar lai, sequences of genes encoding for proteins that resemble mammalian proteins involved in homeostasis (such as platelet activating factor acetylhydrolase, type A domains of von Willebrand factor, and paraoxanase) have been described. The authors of that study hypothesized that the combination of proteases and the proteins involved in homeostasis could be involved in the genesis of the hemorrhagic lesions [48]. Such genomic studies will certainly enhance our understanding of the molecular mechanisms of leptospiral pathogenesis and contribute to the development of novel vaccines.

\section{Pathophysiology of Pulmonary Leptospirosis: Experimental Studies}

Animal models have been used to highlight the pathogenetic events leading to alveolar hemorrhage in leptospirosis. Several authors have used the model of intraperitoneal inoculation of Leptospira in rodents (guinea pigs, hamsters and mice) and monkeys, resulting in hemorrhagic lesions in various organs, including the lungs [49-52].

More severe lung lesions were achieved using the pathogenic Leptospira interrogans serovars copenhageni or icterohaemorrhagiae. In some studies, authors described that the inoculated bacteria was isolated from blood cultures of patients suffering from the severe pulmonary form of leptospirosis [53]. The sequence of events culminating in lung hemorrhage is mostly similar in the available models [49,5456]. The studies describe initial capillary congestion followed by endothelial injury and increasing larger areas of parenchymal hemorrhages within a few days (5-10) after inoculation. Foci of lung hemorrhage in a smaller proportion of animals as soon as 2-6 hours after leptospires inoculation were described [54]. Endothelial cell alterations such as swelling and necrosis, presence of fibrin thrombi and mild to moderate alveolar septa inflammation are consistently reported.

Miller et al, in 1974, described in details the acute lesions occurring in the lungs 48 hours after Leptospira interrogans serotype icterohaemorrhagiae intraperitoneal inoculation [54]. After this period all animals presented pulmonary hemorrhage, but the number of leptospires isolated from the lungs was very low when compared to the liver, and no leptospires could be detected by histochemical methods in lung tissue. At optical level, areas of intra-alveolar hemorrhage, with increased numbers of leukocytes in alveolar septa and sporadic fibrin deposition was observed. At ultrastructural level, the authors observed that the capillary endothelium was often detached from the basement membrane, as well as the presence of endothelial and epithelial blebs. Curiously, intercellular junctions appeared to be intact. De Brito et al, in 1979, confirmed similar findings, describing optical and ultrastructural aspects of lung, kidney and diaphragm capillaries of guinea pigs infected with Leptospira icterohaemorrhagiae [49]. Many blebs in the endothelial cells were also observed. The authors further described the presence of platelet and leukocyte thrombi in the alveolar capillaries. These alterations were ascribed as non-specific reactions, occurring in many other situations of severe damage to lung vessels. Similarly, a paucity of microorganisms was observed at the site of the lesions in the latter study. Both studies hypothesized a "toxic genesis" for the vascular damage, further postulating that primary alterations of lung capillaries permeability should be the initiating event in the pathogenesis of pulmonary hemorrhage in leptospirosis [49,54].

Interestingly, in animal models simulating the so-called "benign-leptospirosis", the lung seems to be spared or little affected. Marshall et al., in a model of "benign" leptospirosis caused by Leptospira interrrogans serovars balcanica and tarassovi in monkeys, could not demonstrate pulmonary histological alterations, while inflammatory lesions were present in the meninges, kidneys and femoral musculature [52].

Although leptospiral antigen has been detected in human lung tissue from patients that died of severe forms of leptospirosis, suggesting a local effect of the bacteria at endothelial level, this is still a controversial issue. Several animal studies have shown that, in comparison to other organs such as the kidney or liver, Leptospira are scantly isolated in the lungs [49,53]. Recently, Nally et al. obtained isolates of Leptospira serovar copenhageni from three patients that died of pulmonary hemorrhage, and inoculated the material in guinea pigs. Animals developed multi-focal areas of hemorrhage in the lungs and intestinal mesenteric surfaces, but not in the liver, kidneys or spleen. In the lungs, alveolar hemorrhage, mild to moderate numbers of mononuclear cells and neutrophils, and rare leptospires (detected by means of immunohistochemistry and electron microscopy), were observed. Interestingly, many leptospires were detected in the liver and kidneys of the animals, but without associated hemorrhagic phenomena. The authors suggested that a high concentration of bacteria in a determined tissue does not necessarily leads to hemorrhage [53]. However, Pereira et al, using marmoset monkeys, demonstrated with immunofluorescence the presence of amorphous leptospires antigens in lung tissue, using a monoclonal antibody. 
Interestingly, intact leptospires could not be detected in the lungs using a rabbit polyclonal antibody. In this study, the authors did not compare the relative amount of antigen found in each organ [57]. It is possible that the differences in antigen detection in the different studies might be mainly attributed to the kind of antigen detection used.

Another controversial point in relation to pulmonary hemorrhage in leptospirosis is whether it could be associated with Disseminated Intravascular Coagulation (DIC) or not. Silva et al, in 1995, described the sequence of events culminating in multi-organ hemorrhage after intraperitoneal inoculation of a virulent strain of Leptospira interrogans serovar copenhageni in guinea pigs [56]. They suggested that Disseminated Intravascular Coagulation is responsible for the hemorrhagic phenomena that increasingly occur as the disease runs its course. They credited this fact to the observed increased prothrombin and pro-thromboplastin times, platelet depletion, and reduced fibrinogen levels observed in the animals. Also, laboratorial signs of DIC were observed in the guinea pig model described by Higgins and Cousineau [55]. However, Nally et al. could not demonstrate marked alterations in platelet numbers or other laboratory signs of DIC in a model of severe pulmonary leptospirosis, also in guinea pigs [53]. In humans, it is currently believed that the hemorrhagic phenomena of leptospirosis are not a result of DIC [37].

Finally, few studies addressed the immunological aspects of the disease. The inflammatory response in leptospirosis is disproportionally mild in relation to the amount of vascular damage in the more severe cases. Nally et al. hypothesized that Leptospira employs a specific mechanism to diminish the magnitude of host inflammatory response. These authors demonstrated the deposition of IgG, IgA and C3 along the alveolar basement membrane and proposed that an autoimmune process could be involved in the mechanisms of severe pulmonary hemorrhage [53]. Pereira et al. described that young $\mathrm{C} 3 \mathrm{H} / \mathrm{HeJ}$ mice were highly susceptible to lethal Leptospira infections. Adult mice, over four weeks old, became more resistant. These authors also demonstrated that CD4 and CD8 depleted mice show more extensive areas of lung hemorrhage associated with vasculitis, sustained and amplified lung and kidney inflammation, and persistence of severe kidney acute tubular necrosis. These findings suggest a role for these cells in the immunopathology of leptospirosis [51].

\section{References}

1. Vinetz J.M. Leptospirosis. Curr Opin Infect Dis 2001;14:527-38.

2. Sarkar U., Nascimento S.F., Barbosa R., et al. Population-based case-control investigation of risk factors for leptospirosis during an urban epidemic. Am J Trop Med Hyg 2002;66:605-10.

3. Martinez Garcia M.A., de Diego Damia A., Menendez Villanueva R., Lopez Hontagas J.L. Pulmonary involvement in leptospirosis. Eur J Clin Microbiol Infect Dis 2000;19:471-4.

4. Luks A.M., Lakshminarayanan S., Hirschmann J.V. Leptospirosis presenting as diffuse alveolar hemorrhage: case report and literature review. Chest 2003;123:639-43.

5. Bharti A.R., Nally J.E., Ricaldi J.N., et al. Leptospirosis: a zoonotic disease of global importance. Lancet Infect Dis 2003;3:757-71.
6. Vinetz J.M., Glass G.E., Flexner C.E., et al. Sporadic urban leptospirosis. Ann Intern Med 1996;125:794-8.

7. Carvalho C.R.R., Bethlem E.P. Pulmonary complications of leptospirosis. Clin Chest Med 2002;23:469-78.

8. Zaki S.R., Shieh W.J. Leptospirosis associated with outbreak of acute febrile illness and pulmonary hemorrhage, Nicaragua, 1995. The Epidemic Working Group at Ministry of Health in Nicaragua. Lancet 1996;347:535-6.

9. Sehgal S.C., Vijayachari P., Smythe L.D., et al. Lai-like leptospira from the Andaman Islands. Indian J Med Res 2000;112:135-9.

10. Yersin C., Bovet P., Merien F., et al. Pulmonary hemorrhage as a predominant cause of death in leptospirosis in Seychelles. Trans R Soc Trop Med Hyg 2000;94:71-6.

11. Simpson F.G., Green K.A., Haug G.J., Brookes D.L. Leptospirosis associated with severe pulmonary hemorrhage in Far North Queensland. Med J Aust 1998;169:151-3.

12. Divate S.A., Chaturvedi R., Jadhav N.N., Vaideeswar P. Leptospirosis associated with diffuse alveolar hemorrhage. J Postgrad Med 2002;48:131-2.

13. Vijayachari P., Sehgal S.C., Goris M.G., et al. Leptospira interrogans serovar Valbuzzi: a cause of severe pulmonary hemorrhages in the Andaman Islands. J Med Microbiol 2003;52:913-8.

14. O’Neil K.M., Rickman L.S., Lazarus A.A. Pulmonary manifestations of leptospirosis. Rev Infect Dis 1991;13:705-9.

15. Seguro E.R., Ganoza C.A., Campos K., et al. Clinical spectrum of pulmonary involvement in leptospirosis in a region of endemicity, with quantification of leptospiral burden. Clin Infect Dis 2005; 40 :343-51.

16. Goncalves A.J., de Carvalho J.E., Guedes e Silva J.B., et al. Hemoptysis and the adult respiratory distress syndrome as the causes of death in leptospirosis. Changes in the clinical and anatomicopathological patterns. Rev Soc Bras Med Trop 1992;25:261-70.

17. Chee H.D., Ossenkoppele G.J., Bronsveld W., Thijs L.G. Adult respiratory distress syndrome in leptospira icterohaemorrhagiae infection. Intensive Care Med 1985;11:254-6.

18. Levett P.N. Leptospirosis. Clin Microbiol Rev 2001;14:296-326.

19. Pai N.D., Adhikari P.M. Haemorrhagic pneumonitis: A rare presentation of leptospirosis. J Postgrad Med 2001;47:35-6.

20. Teglia O.F., Battagliotti C., Villavicencio R.L., Cunha B.A. Leptospiral pneumonia. Chest 1995;108:874-5.

21. Wang C.N., John L., Chang T.F., et al. Studies on anicteric leptospirosis. I. Clinical manifestations and antibiotic therapy. Chin Med J Engl 1965;84:283-91.

22. Lomar A.V., Diament D., Torres J.R. Leptospirosis in Latin America. Infect Dis Clin North Am 2000;14:23-39.

23. Silva J.J., Dalston M.O., Carvalho J.E., et al. Clinicopathological and immunohistochemical features of the severe pulmonary form of leptospirosis. Rev Soc Bras Med Trop 2002;35:395-9.

24. Edwards C.N., Nicholson G.D., Everard C.O. Thrombocytopenia in leptospirosis. Am J Trop Med Hyg 1982;31:827-9.

25. Nicodemo A.C., Del Negro G., Amato Neto V. Thrombocytopenia and leptospirosis. Rev Inst Med Trop Sao Paulo 1990;32:252-9.

26. Seijo A., Coto H., San Juan J., et al. Lethal leptospiral pulmonary hemorrhage: an emerging disease in Buenos Aires, Argentina. Emerg Infect Dis 2002;8:1004-5.

27. Yersin C., Bovet P., Merien F., et al. Human leptospirosis in the Seychelles (Indian Ocean): a population-based study. Am J Trop Med Hyg 1998;59:933-40.

28. Park S.K., Lee S.H., Rhee Y.K., et al. Leptospirosis in Chonbuk Province of Korea in 1987: a study of 93 patients. Am J Trop Med Hyg 1989;41:345-51.

29. Dupont H., Dupont-Perdrizet D., Perie J.L., et al. Leptospirosis: prognostic factors associated with mortality. Clin Infect Dis 1997;25:720-4.

30. Donaghy M., Rees A.J. Cigarette smoking and lung hemorrhage in glomerulonephritis caused by autoantibodies to glomerular basement membrane. Lancet 1983;2:1390-3. 
31. Marotto P.C., Nascimento C.M., Eluf-Neto J., et al. Acute lung injury in leptospirosis: clinical and laboratory features, outcome, and factors associated with mortality. Clin Infect Dis 1999;29:1561-3.

32. Kiatboonsri S., Vathesatogit P., Charoenpan P. Adult respiratory distress syndrome in Thai medical patients. Southeast Asian J Trop Med Public Health 1995;26:774-80.

33. Im J.G., Yeon K.M., Han M.C., et al. Leptospirosis of the lung: radiographic findings in 58 patients. Am J Roentgenol 1989;152:955-9.

34. Marchiori E., Muller N.L. Leptospirosis of the lung: high-resolution computed tomography findings in five patients. J Thorac Imaging 2002;17:151-3.

35. Arean V.M. The pathologic anatomy and pathogenesis of fatal human leptospirosis (Weil's disease). Am J Pathol 1962;40:393-423.

36. Zaltzman M., Kallenbach J.M., Goss G.D., et al. Adult respiratory distress syndrome in Leptospira canicola infection. Br Med J (Clin Res Ed) 1981;283:519-20.

37. Nicodemo A.C., Duarte M.I., Alves V.A., et al. Lung lesions in human leptospirosis: microscopic, immunohistochemical, and ultrastructural features related to thrombocytopenia. Am J Trop Med Hyg 1997;56:181-7.

38. Barocchi M.A., Ko A.I., Reis M.G., et al. Rapid translocation of polarized MDCK cell monolayers by Leptospira interrogans, an invasive but nonintracellular pathogen. Infect Immun 2002;70:6926-32.

39. Cullen P.A., Haake D.A., Adler B. Outer membrane proteins of pathogenic spirochetes. FEMS Microbiol Rev 2004;28:291-318.

40. Vinh T., Adler B., Faine S. Glycolipoprotein cytotoxin from Leptospira interrogans serovar copenhageni. J Gen Microbiol 1986;132:111-23.

41. Lee S.H., Kim S., Park S.C., Kim M.J. Cytotoxic activities of Leptospira interrogans hemolysin $\mathrm{SphH}$ as a poreforming protein on mammalian cells. Infect Immun 2002; 70:315-22.

42. De-Souza L., Koury M.C. Chemical and biological properties of endotoxin from Leptospira interrogans serovars canicola and icterohaemorrhagiae. Braz J Med Biol Res 1992; 25:467-75.

43. Dobrina A., Nardon E., Vecile E., et al. Leptospira icterohemorrhagiae and leptospire peptidolgycans induce endothelial cell adhesiveness for polymorphonuclear leukocytes. Infect Immun 1995;63:2995-9.

44. Estavoyer J.M., Racadot E., Couetdic G., et al. Tumor necrosis factor in patients with leptospirosis. Rev Infect Dis 1991;13:1245-6.
45. Werts C., Tapping R.I., Mathison J.C., et al. Leptospiral lipopolysaccharide activates cells through a TLR2-dependent mechanism. Nat Immunol 2001;2:346-52.

46. Abdulkader R.C., Daher E.F., Camargo E.D., et al. Leptospirosis severity may be associated with the intensity of humoral immune response. Rev Inst Med Trop Sao Paulo 2002;44:79-83.

47. Nascimento A.L., Ko A.I., Martins E.A., et al. Comparative genomics of two Leptospira interrogans serovars reveals novel insights into physiology and pathogenesis. J Bacteriol 2004; $186: 2164-72$.

48. Ren S.X., Fu G., Jiang X.G., et al. Unique physiological and pathogenic features of Leptospira interrogans revealed by wholegenome sequencing. Nature 2003;422:888-93.

49. De Brito T., Bohm G.M., Yasuda P.H. Vascular damage in acute experimental leptospirosis of the guinea pig. J Pathol 1979;128:177-82.

50. Oliva R., Infante J.F., Gonzalez M., et al. Pathologic-clinical characterization of leptospirosis in a golden Syrian hamster model. Arch Med Res 1994;25:165-70.

51. Pereira M.M., Andrade J., Marchevsky R.S., Ribeiro dos Santos R. Morphological characterization of lung and kidney lesions in $\mathrm{C} 3 \mathrm{H} / \mathrm{HeJ}$ mice infected with Leptospira interrogans serovar icterohaemorrhagiae: defect of CD4+ and CD8+ T-cells are prognosticators of the disease progression. Exp Toxicol Pathol 1998;50:191-8.

52. Marshall R.B., Baskerville A., Hambleton P., Adams G.D. Benign leptospirosis: the pathology of experimental infection of monkeys with Leptospira interrogans serovars balcanica and tarassovi. Br J Exp Pathol 1980;61:124-31.

53. Nally J.E., Chantranuwat C., Wu X.Y., et al. Alveolar septal deposition of immunoglobulin and complement parallels pulmonary hemorrhage in a guinea pig model of severe pulmonary leptospirosis. Am J Pathol 2004;164:1115-27.

54. Miller N.G., Allen J.E., Wilson R.B. The pathogenesis of hemorrhage in the lung of the hamster during acute leptospirosis. Med Microbiol Immunol (Berl) 1974;160:269-78.

55. Higgins R., Cousineau G. The pathogenesis of leptospirosis I. Hemorrhages in experimental leptospirosis in guinea pigs. Can J Comp Med 1977;41:174-81.

56. da Silva J.J., Netto B.A., Lilembaum W., et al. The hemorrhagic syndrome of leptospirosis: an experimental study in guinea pigs. Rev Soc Bras Med Trop 1995;28:169-77.

57. Pereira M.M., Da Silva J.J., Pinto M.A., et al. Experimental leptospirosis in marmoset monkeys (Callithrix jacchus): a new model for studies of severe pulmonary leptospirosis. Am J Trop Med Hyg 2005;72:13-20. 